\title{
Two Point Fixation in Zygomatic Complex Fractures
}

\author{
Sourav Sharma ${ }^{1 *}$ and Vandana $D^{2}$ \\ ${ }^{1}$ Oral and maxillofacial surgeon. Superspeciality Dental and Maxillofacial centre. Jammu. J\&K. India. \\ ${ }^{2}$ Superspeciality Dental and Maxillofacial centre. Jammu. J\&K. India.
}

\begin{abstract}
Zygomatic bone along with its adjacent articulating bones is known as zygomatic complex. The zygomatic complex fractures are one of the frequently occurring maxillofacial injuries due to its prominence and facial contour. Personal altercations, Assaults, Road traffic accidents are the common causes leading to fracture of the zygomatic bone. The zygomatico-maxillary complex is one of the principle buttress of the face and helps in transmitting the occlusal forces to the skull base.Fracture of zygomatic bone may lead to aesthetic and functional deformity. There are various surgical techniques and approaches to reduce and fix the fractured zygomatic complex. Expertise of the Maxillofacial surgeon and thorough anatomical knowledge is of advantage in managing these fractures.
\end{abstract}

Keywords: Mini Plates, Open Reduction and Internal Fixation, Surgical Approach, Two Pont Fixation.

\section{Introduction}

Zygomatic complex fractures are common maxillofacial inuries.It is always challenging for maxillofacial surgeons who are specialized in treating this part of the human body to achieve the lost functions and regain proper facial contour.

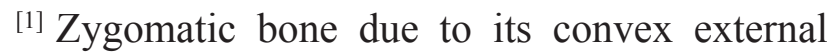
surface provides prominence to the cheek region. Lateral prominence and articulation of zygoma with adjacent facial bones causes this bone to be frequently fractured. ${ }^{[2,3]}$ Proximity of the zygomatic bone with the orbit and maxilla often leads to ocular or visual disturbances and disturbed occlusion. This necessitates surgical intervention in order to achieve satisfactory stability and best post operative results. Closed reduction has been found to be of no use in providing stability and fracture reduction in zygomatic complex fractures. ${ }^{[4]}$

The fractured zygoma biomechanically has six possible directions of motion: rotation around $\mathrm{X}, \mathrm{Y}$ and $\mathrm{Z}$ axis; translation across $\mathrm{X}, \mathrm{Y}$ and $\mathrm{Z}$ axis. Wire fixation of zygomatic complex fracture has shown unsatisfactory results in terms of accurate reduction and fixation of small fractured fragments. ${ }^{[5,6]}$ Mini plates have revolutionized the standards of management of maxillofacial fractures by providing rigid internal fixation and ultimately better stability and less complications. Zygomatic bone is attached to adjacent bones and has four suture lines; which are the areas of anatomical weakness. Depending upon the velocity and impact of the trauma; one or many of these sutures are involved in the fracture. The topic of controversy here has been the amount of fixation which is required to precisely stabilize and fix the fractured zygomatic complex. We present a report of patient with zygomatic complex fracture who was treated by fixation of mini plates at infra orbital rim and frontozygomatic suture region.

\section{Case Report:}

A 40 Year old male reported to our centre with history of road traffic accident three days back. Patient had restricted mouth opening, depressed zygomatic bone and paresthesia of the infra orbital nerve. Radographic investigations revealed fractured zygomatic complex. Opthalmic opinion was taken .Treatment was explained to the patient and informed written consent was obtained prior to surgery (fig 1).

Keen's intraoral technique was used to elevate and reduce the displaced zygomatic complex. 
A small $1 \mathrm{~cm}$ incision in the mucobuccal fold beneath the zygomaticomaxillary buttress was placed. Rowe's zygomatic elevator was inserted behind the infratemporal surface of the zygoma and bone was reduced into correct anatomical location. Head of the patient should be held firmly by the assisting surgeon during this maneuver.

Local anaesthesia with adrenaline 1:80,000 was infiltrated and lateral eyebrow incision approximately $1.5 \mathrm{~cm}$ in length was placed over fronto-zygomatic suture region (fig 2). Skin over the supra orbital rim region should be supported using two fingers. Periosteal elevator should be used while drilling to protect the eyeball from any injury.

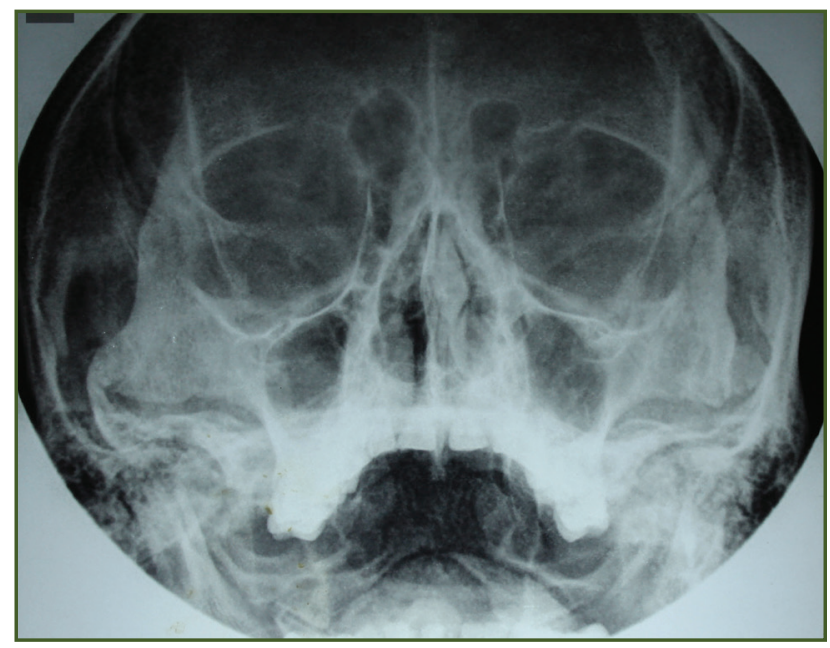

Fig.1: Pns View Showing Left Zmc Fracture.

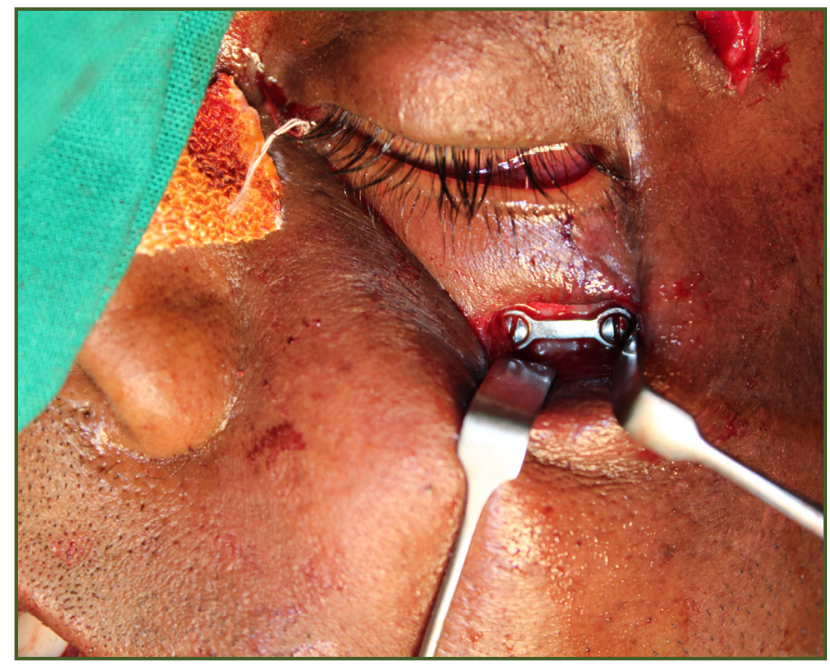

Fig. 3: Mini Plate At Infra Orbital Rim.

Annals of Applied Bio-Sciences, Vol. 4; Issue 1: 2017
Infraorbital incision was then placed and fracture site was exposed and reduction was achieved. First hole should always be drilled in the unstable segment of the fracture. Finally, all the screws were tightened (fig 3). Wound was closed in layers using 3-0 vicryl and 5-0 ethilon sutures. Post operative medications were advised for atleast a week. Suture removal was done on $8^{\text {th }}$ postoperative day. Healing was uneventful .Post operative PNS view was taken after surgery (fig 4). Patient was on periodic follow up and Infra orbital paresthesia was resolved. Patient had an almost imperceptible scar.

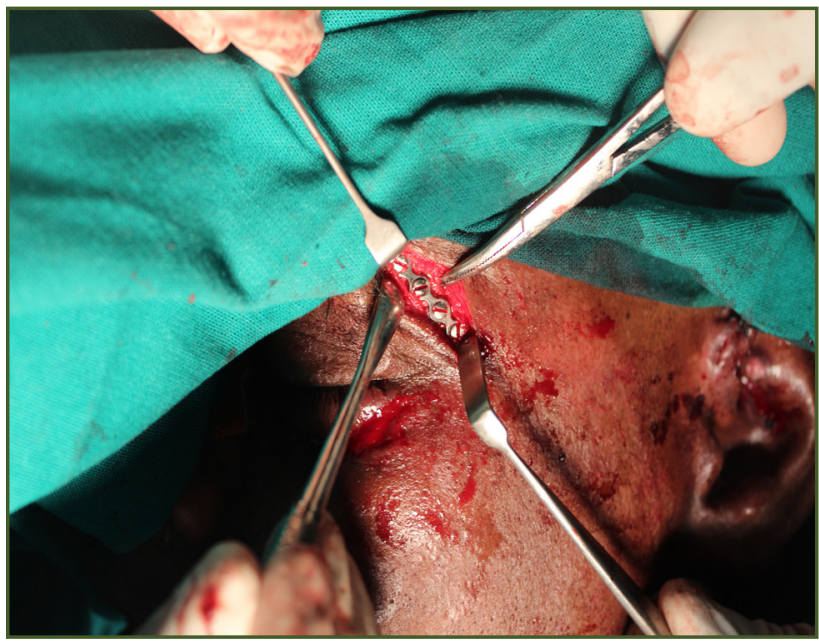

Fig. 2: Mini Plate Fixation At Fronto Zygomatic Suture

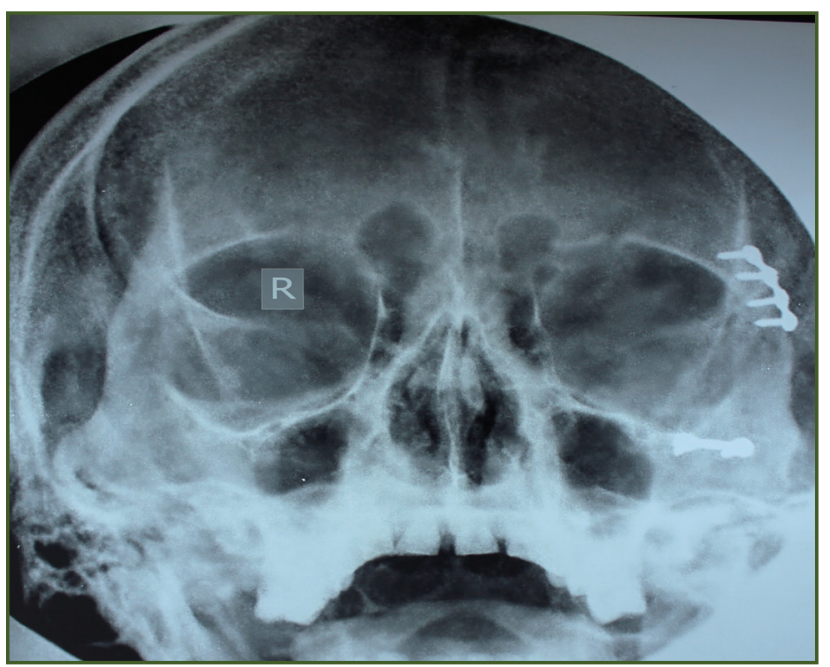

Fig. 4: Post Op Pns View Showing Two Point Fixation and Stabilization.

e-ISSN: 2349-6991; p-ISSN: 2455-0396 


\section{Discussion}

Facial asymmetry due to facial trauma may lead to mental agony and social discomfort. Progressive advent and gradual increase in number of high speed vehicles has led to increased cases of facial trauma. The integrity of the zygomatic complex is critical in maintaining normal facial width and cheek prominence. Zygomatic bone alone rarely gets fractured; its articulating surfaces: maxilla, temporal, frontal and sphenoid are also involved. It serves as the buttress between the face and the skull which absorbs the distortion forces and gets fractured at the sutures; thereby reducing the impact on the brain.

Peter B Grey et $\mathrm{a}^{\left[{ }^{7]}\right.}$ advocated ophthalmic opinion is mandatory in most of the zygomatic complex fractures; as delayed retrobulbar haemorrhage may lead to blindness. Infra orbital and zygomatico frontal suture approach was used to treat the displaced zygomatic complex fractures. ${ }^{[8]}$ Six mm screws can be safely placed above the frontozygomatic suture. Infra orbital rim incision provided adequate exposure of the fracture site; compressed infra orbital nerve was released from the fractured fragments. Fractured bony segments were accurately aligned and fixed using mini plate. Studies have also shown higher incidence of recovery of infra orbital nerve paresthesia using mini plate osteosynthesis.

\section{Conclusion}

We conclude that two point fixation in Zygomatic complex fractures yields satisfactory post operative stability and aesthetics in addition to resolution of infra orbital paresthesia. Severely displaced zygomatic complex fractures may require additional points of fixation.

\section{Reference}

1. Zingg $\mathrm{M}$, Laedrach $\mathrm{K}$, Chen $\mathrm{J}$ et al.Classification and treatment of zygomatic fractures:A review of 1025 cases.J Oral Maxillofac Surg 1992; 50:778-790.

2. Covington D.S, D.J Wainwright, J.F Teichgraeber and D.H Parks.Changing patterns in the epidemiology and treatment of zygoma fractures:10 year review. J Trauma 1994;37(2):243-248.

3. Poswillo D.Reduction of the fractured malar by a traction hook. British Journal of Oral Surgery1976;14:76-79.

4. Kovacs A.F, Ghahremani M. Minimization of zygomatic complex fracture treatment. J Oral Maxillofac Surg 2001;30(5):380-383.

5. Lund K .Fractures of the zygoma:a follow up study on 62 patients. J Oral Surg 1971;29:557-560.

6. Pozatek ZW, Kaban LB, Guralnick WC.Fractures of the zygomatic complex:an evaluation of surgical management with special emphasis on the eyebrow approach. J Oral Surg 1973;31:141-148.

7. Gray B.P,Leen MM and Loftus M.J.Late retrobulbar hemorrhage and blindness following malar fracture complicated by factor XI deficiency.Journal of Oral and Maxillofacial Surgery 1993;51:699-702.

8. Olate,Monteiro L, Renato S, Roger W F,Marcio M.Surgical Approaches and Fixation Patterns in Zygomatic Complex Fractures. Journal of Craniofacial Surgery2010; 21(4): 1213-1217.

*Corresponding author:

SOURAV SHARMA, 102/A. Main Road Roop Nagar. Near Janipur Police Station. Jammu. J\&K.180013. India

Email: dr.souravsurgeon@gmail.com 